\title{
3T Intraoperative MRI for Management of Pediatric CNS Neoplasms
}

\author{
(D) A.F. Choudhri, P. Klimo Jr, T.S. Auschwitz, M.T. Whitehead, and F.A. Boop
}

\begin{abstract}
BACKGROUND AND PURPOSE: High-field-strength intraoperative MR imaging has emerged as a powerful adjunct for resection of brain tumors. However, its exact role has not been firmly established. We sought to determine the impact of 3T-intraoperative MRI on the surgical management of childhood CNS tumors.
\end{abstract}

MATERIALS AND METHODS: We evaluated patient data from a single academic children's hospital during a consecutive 24-month period after installation of a 3T-intraoperative MRI. Tumor location, histology, surgical approach, operating room time, presence and volume of residual tumor, need for tumor and non-tumor-related reoperation, and anesthesia- and MR imaging-related complications were evaluated. Comparison with pre-intraoperative MRI controls was performed.

RESULTS: One hundred ninety-four patients underwent intraoperative MRI- guided surgery. Of these, 168 were 18 years or younger (mean, $8.9 \pm 5.0$ years; 108 males $/ 60$ females). There were 65 posterior fossa tumors. The most common tumors were pilocytic astrocytoma $(n=$ $31,19 \%)$, low-grade glioma ( $n=31,19 \%$ ), and medulloblastoma $(n=20,12 \%)$. An average of 1.2 scanning sessions was performed per patient (maximum, 3). There were no MR imaging-related safety issues. Additional tumor was resected after scanning in $21 \%$ of patients. Among patients with a preoperative goal of gross total resection, 93\% achieved this goal. The 30-day reoperation rate was $<1 \%$ ( $n=1$ ), and no patient required additional postoperative MR imaging during the same hospital stay.

CONCLUSIONS: Intraoperative MRI is safe and increases the likelihood of gross total resection, albeit with increased operating room time, and reduces the need for early reoperation or repeat sedation for postoperative scans in children with brain tumors.

ABBREVIATIONS: FSPGR $=$ fast-spoiled gradient recalled; iMRI $=$ intraoperative MRI

B rain tumors are the second most common type of pediatric cancer, affecting more than 4000 children per year in the United States. ${ }^{1}$ For many tumors, such as ependymoma and medulloblastoma, maximal cytoreductive resection offers the greatest chance for long-term survival. ${ }^{2}$ Intraoperative MR imaging (iMRI) has been proposed as an adjunctive technique to achieve maximal tumor resection while limiting iatrogenic neurologic in-

Received January 30, 2014; accepted after revision May 26

From the Departments of Radiology (A.F.C., M.T.W.), Neurosurgery (A.F.C., P.K., T.S.A., F.A.B.), and Ophthalmology (A.F.C.), University of Tennessee Health Science Center, Memphis, Tennessee; Le Bonheur Neuroscience Institute (A.F.C., P.K., M.T.W., F.A.B.), Le Bonheur Children's Hospital, Memphis, Tennessee; SemmesMurphey Neurologic and Spine Institute (P.K., F.A.B.), Memphis, Tennessee; Division of Neurosurgery (P.K., F.A.B.), and Department of Surgery, St. Jude Children's Hospital, Memphis, Tennessee; and Department of Radiology (M.T.W.), Children's National Medical Center, Washington, DC.

This work was supported, in part, by a grant from Le Bonheur Children's Hospital and Children's Research Foundation to A.F.C.

Please address correspondence to Asim F. Choudhri, MD, Department of Radiology, Le Bonheur Children's Hospital, 848 Adams Ave-G216, Memphis, TN 38103;

e-mail: achoudhri@uthsc.edu; @AsimChoudhriMD

http://dx.doi.org/10.3174/ajnr.A4040 jury. ${ }^{3-11}$ However, the benefits of this expensive technology have not been uniformly demonstrated in the literature. ${ }^{11-14}$ With respect to high-field-strength iMRI, typically defined as $1.5 \mathrm{~T}$ or higher, its impact on operating room and anesthesia time and the incidence of MR imaging-related complications, particularly in the pediatric population, have been the topic of limited publications. ${ }^{3,15-17}$ We performed a retrospective review of our 24month experience with a 3T-iMRI at a tertiary care children's hospital to determine the impact of this tool on surgical planning, workflow, and patient outcomes. Comparison was made with a cohort of pediatric patients with brain tumor at our institution from before installation of the iMRI scanner.

\section{MATERIALS AND METHODS}

This Health Insurance Portability and Accountability Act-compliant retrospective study was performed after institutional review board approval. We evaluated 3T-iMRI patient data for a consecutive 24-month period (February 2011 to February 2013) from a single academic children's hospital, identifying patients 18 
years or younger who underwent iMRI-guided resection. Preoperative, intraoperative, MR imaging-related, and postoperative parameters and outcomes were recorded.

Preoperative parameters evaluated included the age, sex, number of prior tumor resections or biopsies, and the presence of a preoperative shunt. Intraoperative parameters evaluated included operating room entry time, time of initial skin incision and skin closure, operating room exit time, patient position, surgical approach, estimated blood loss, and anesthesia-related complications. We determined whether frameless stereotactic navigation was used and whether this was performed by using scans obtained in the operating room or before arrival in the operating room.

MR imaging parameters evaluated included the number of intraoperative scans, the time required to place the MR imaging coils and bring the scanner into the room, the number of scoutlocalizers required, the total amount of intravenous contrast administered, and any MR imaging-related complications. The presence of residual tumor on MR imaging scans was also documented. The extent of resection was graded as gross total resection if $>98 \%$ of the tumor was resected, near-total if $>90 \%$ was resected, and subtotal if $<90 \%$ was resected.

Postoperative parameters included tumor histology, length of stay (intensive care, total hospital admission), disposition at time of discharge (home versus other facility), 7- and 30-day reoperation rates, mortality, and surgical site complications.

\section{MR Imaging Scanner and Imaging Protocol}

iMRI scans were performed with a 3T unit (Magnetom Verio 3T; Siemens, Erlangen, Germany), which was brought into the operating room via a ceiling-mounted track system (IMRIS-neuro; IMRIS, Winnipeg, Manitoba, Canada). A 2-part split-array 8-channel head coil (HC-300; IMRIS-neuro) was used, with one 4-channel component of the coil placed inferior to the patient's head and one 4-channel component placed superior to the patient's head.

For the first 12 months, the routine intraoperative scanning protocol included axial and coronal T1-weighted images before and after contrast administration and an axial T2-weighted sequence and diffusion-weighted imaging. Additional planes were used in select cases as needed, most often sagittal T1 postcontrast for posterior fossa tumors. After 12 months, the protocol was revised to include axial 3D fast-spoiled gradient recalled (FSPGR) images before and after contrast administration, an axial T2weighted image, and axial diffusion-weighted images. The axial T2-weighted image was obtained immediately after contrast administration to allow a contrast agent infusion delay before the FSPGR images. Subtraction imaging was performed between the pre- and postcontrast FSPGR sequence, and FSPGR reformats were performed in the sagittal and coronal planes.

Imaging studies were interpreted at the time of scanning by 1 of 2 fellowship-trained board-certified neuroradiologists (A.F.C. or M.T.W.) and were reviewed in direct consultation with the attending neurosurgeon (F.A.B. or P.K.).

\section{Historical Comparison}

A hospital neuro-oncology data base was queried to evaluate the incidence of tumor-related reoperations at our hospital in the time before institution of the iMRI program.
Table 1: Tumor location

\begin{tabular}{lcc}
\hline & No. & (\%) \\
\hline Supratentorial (NOS) & 64 & 38 \\
Infratentorial (not brain stem) & 43 & 26 \\
Brain stem & 22 & 13 \\
Midbrain/pineal & 13 & 8 \\
Third ventricular/suprasellar & 12 & 7 \\
Deep gray nuclei & 9 & 5 \\
Cervical spine/skull base & 5 & 3 \\
\hline
\end{tabular}

Note:-NOS indicates not otherwise specified.

Table 2: Tumor histology

\begin{tabular}{lrr}
\multicolumn{1}{c}{ Histology } & No. & (\%) \\
\hline Pilocytic astrocytoma & 31 & 19 \\
Low-grade glioma & 31 & 19 \\
Medulloblastoma/PNET & 20 & 12 \\
Ependymoma & 15 & 9 \\
High-grade glioma & 14 & 8 \\
Ganglioglioma & 10 & 6 \\
Other & 47 & 28 \\
\hline
\end{tabular}

Note:-PNET indicates primitive neuroectodermal tumor.

\section{Data Collection and Statistics}

Data were stored on a spreadsheet (Excel 2011; Microsoft, Redmond, Washington). Data were analyzed by using Excel and SPSS, Version 20 (IBM, Armonk, New York). Discrete variables were compared by using the Fisher exact test. Continuous variables were compared by using the Kruskall-Wallis test, with a nonparametric posttest, or a Student $t$ test when data followed a Gaussian distribution. A $P$ value $<.05$ was considered significant.

\section{RESULTS}

\section{Patient Demographics}

One hundred ninety-four patients underwent iMRI-guided surgery at our institution in the 24 months after installation. Of these, $168(87 \%)$ were 18 years or younger (mean, $8.9 \pm 5.0$; range, $1.2-18.5$ years; median, 8.0 years). There were 60 females and 108 males. Sixty-five (39\%) tumors were located in the posterior fossa (Table 1). The most common tumors were pilocytic astrocytoma ( $n=31,19 \%)$, low-grade glioma $(n=31,19 \%)$, and medulloblastoma $(n=20,12 \%)$ (Table 2). Prior tumor resection was performed in 60 patients (38\%), and $34(21 \%)$ had pre-existing CSF shunts. The prone position was used in 79 patients; supine, in 70; and lateral decubitus, in 19.

\section{MR Imaging Scans}

Within the pediatric subset of patients, an average of 1.2 intraoperative scanning sessions per patient was performed (median, 1; maximum, 3 scan sessions). There were no MR imaging-related safety issues. Intraoperative gadolinium was administered in 152 patients (90\%). For patients receiving gadolinium for the intraoperative scan, an average of 1.1 doses was administered (median, 1 ; range, 1-3). The dose for the first 2 administrations was 0.1 $\mathrm{mmol} / \mathrm{kg}$ of gadopentetate dimeglumine. When required, the third administration was a half-dose $(0.05-\mathrm{mmol} / \mathrm{kg}$ gadopentetate dimeglumine).

\section{Operating Room Workflow}

The mean time in the operating room, from the patient entering the room to exiting the room, was $428 \pm 143$ minutes (range, 
158-958 minutes; median, 415 minutes) (Table 3). The time from operating room entry to initial skin incision was $91 \pm 40$ minutes (range, 19-237 minutes; median, 78 minutes).

Thirty-four patients underwent a stereotactic MR imaging scan in the operating room before starting the case, with a time from entering the operating room to initial skin incision of $145 \pm$ 36 minutes (median, 145 minutes). Compared with the 134 pa-

Table 3: Mean time in the operating room ${ }^{\mathrm{a}}$

\begin{tabular}{lccc}
\hline & Total OR Time & $\begin{array}{c}\text { OR Entry } \\
\text { to Skin }\end{array}$ & Skin to Skin \\
\hline All patients & $428 \pm 142(415)$ & $91 \pm 40(78)$ & $316 \pm 130(315)$ \\
Group 1 & $491 \pm 173(469)$ & $145 \pm 36(145)$ & $323 \pm 157(330)$ \\
Group 2 (all pts) & $450 \pm 141(439)$ & $98 \pm 40(82)$ & $331 \pm 129(329)$ \\
Group 2a & $482 \pm 126(483)$ & $75 \pm 19(70)$ & $389 \pm 114(387)$ \\
Group 2b & $415 \pm 120(412)$ & $82 \pm 24(77)$ & $312 \pm 113(302)$ \\
$P$ (gl vs g2) & .08 & $<.0001$ & $\mathrm{NS}(.67)$ \\
$P$ (g2a vs g2b) & .03 & .21 & 0.008 \\
\hline
\end{tabular}

Note:-OR indicates operating room; pts, patients; gl, group 1; g2, group 2; g2a, group 2a; g2b, group 2b; NS, not significant.

a Time is listed in minutes; numbers in parentheses are the median values. Group 1 indicates those with an intraoperative stereotactic scan; group 2, no intraoperative stereotactic scan; group 2a, no stereotactic navigation used; group 2b, stereotactic scan obtained prior to arrival in the operating room. tients who did not have a stereotactic MR imaging scan in the operating room before starting the procedure, which included 98 patients with previously performed stereotactic scans and 36 performed without stereotactic guidance, the mean time from entering the room to initial skin incision was $69 \pm 20$ minutes (median, 63 minutes; $P<.0001)$.

\section{Peri- and Postoperative Outcomes}

The preoperative surgical goal was individualized, primarily depending on the tumor location: gross total resection $(n=112)$, debulking $(n=40)$, biopsy $(n=12)$, and other $(n=4)$.

Additional tumor was resected in 35 (21\%) patients (Fig 1) after scanning. Intraoperatively acquired volumetric images were merged with the stereotactic navigation study in 22 patients to correct for brain shift and guide continuation of resection. Of 112 with a goal of gross total resection, 26 (23\%) had further resection of the lesion, and at the end of the procedure, 104 of these patients (93\%) had gross total resection, with 6 having near-total resection and 2 having subtotal resection. Of 40 patients with a preoperative goal of debulking, 2 had gross total resection, 20 had near-total resection, 16 had subtotal resection, and 2 had biopsy. Estimated
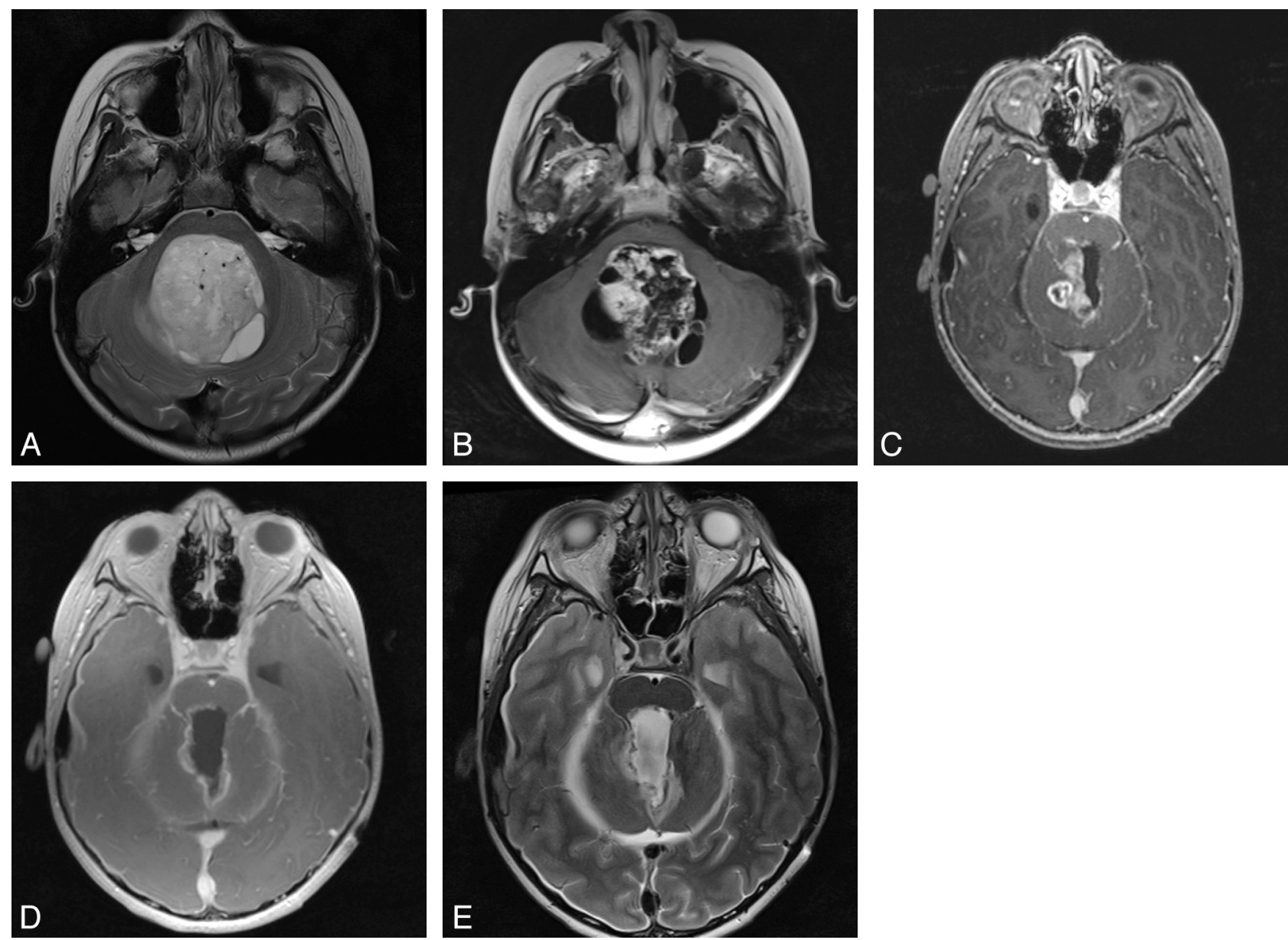

FIG 1. A, Axial T2-weighted image shows a mass in the fourth ventricle in a 9-year-old boy. $B$, Axial T1-weighted image after gadolinium administration shows heterogeneous enhancement within the lesion. C, Axial T1 postgadolinium image from intraoperative MRI shows residual tumor along the right superolateral aspect of the resection cavity. D, Axial TI postgadolinium image from intraoperative MRI after continuation of resection shows removal of the previously seen residual lesion. A rim of enhancement was seen along the margins of the resection cavity; however, no discrete lesion was identified on surgical inspection of the margins. E, Axial T2WI shows a margin of T2 shortening with subjacent edema in the areas of enhancement, a pattern that corresponds to recent use of bipolar electrocautery. On this basis, no further resection was performed. 

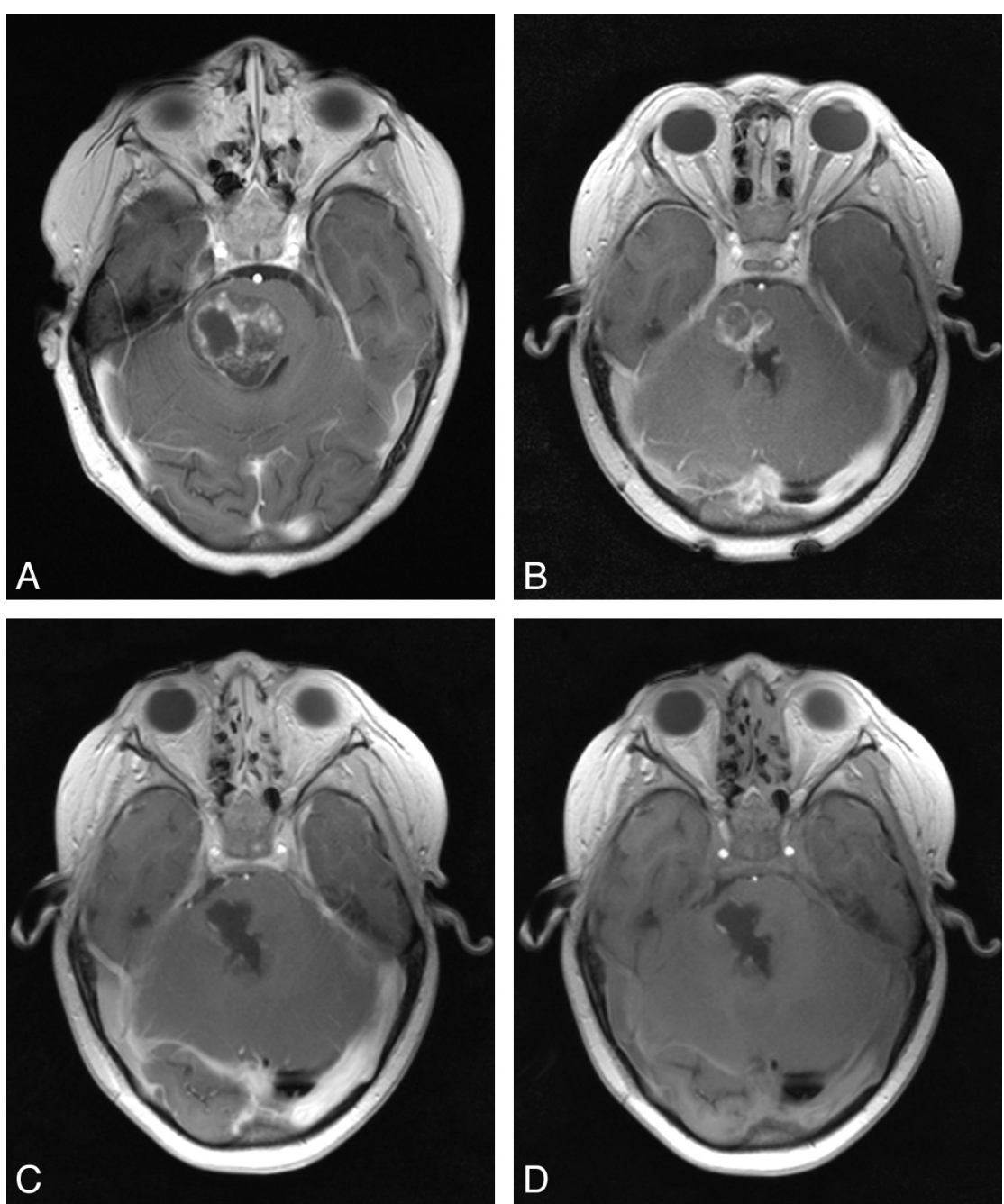

FIG 2. A, Axial TIWI postgadolinium image at the level of the midpons in a 3-year-old girl shows a heterogeneously enhancing mass, which causes near-complete effacement of the fourth ventricle. $B$, Axial TIWI postgadolinium iMRI obtained after initial resection of the lesion shows decreased bulk of the lesion and decreased mass effect on the fourth ventricle. Volumetric $\mathrm{TI}$ images were merged to the stereotactic dataset to guide further resection; this step allowed the procedure to go forward, accounting for intraoperative "brain-shift" from ventricular decompression and tumor debulking. C, Axial TTWI postgadolinium iMRI image after continuation of resection shows several areas of $\mathrm{Tl}$ shortening along the posterior margin of the resection cavity. Review of the resection cavity under an operating microscope showed no macroscopic blood products in this location. $D$, Axial TIWI iMRI after continuation of resection prior to contrast administration shows a similar pattern of $\mathrm{Tl}$ shortening; this represents intrinsic $\mathrm{Tl}$ shortening (presumably related to electrocautery use). Accordingly, there was no abnormal enhancement on the second postcontrast iMRI scan.

blood loss was $141 \pm 137 \mathrm{~mL}$ (range, 10-1000 mL; median, $100 \mathrm{~mL})$.

One hundred fifty-one patients went to the intensive care unit after surgery, and 17 did not. The average intensive care unit length of stay was $2.3 \pm 3.9$ days (range, $0-35$ days; median, 1 day). The total hospital length of stay for all patients was $7.6 \pm 5.3$ days (range, 1-35 days; median, 6 days). Discharge disposition was most commonly to home $(n=99)$. Twenty-nine patients were transferred to a partner institution $(n=29)$ for continued convalescence and others to a hospital-partnered family temporary housing $(n=23)$.

The tumor-related reoperation rate was $0 \%$ at 7 days and $1 \%$ at 30 days ( 1 of 168). The one patient who required reoperation was an 11-year-old boy who had a right frontal lesion originally postulated to be a low-grade lesion. After pathologic determination of a highgrade glioma, the decision was made to further extend the margins to ensure the removal of nonenhancing T2/FLAIR signal abnormality that was originally thought to represent edema. The allcause reoperation rate was $6 \%$ at 7 days $(n=10)$ and $7 \%$ at 30 days $(n=11)$, with reoperations for shunt placement/ revision $(n=6)$, pseudomeningocele $(n=3)$, and intracranial pressure monitor placement $(n=1)$ within the first 7 days and for additional tumor resection $(n=1)$ within the first 30 days.

The 30-day mortality rate after iMRI-guided tumor resection was $0 \%$. At 30 days, 2 patients developed superficial wound infections and a third patient developed a deep infection. There have been no clinical signs of contrast-induced nephrogenic systemic fibrosis in any of the patients.

In the 12 months before instituting the iMRI program, 104 patients underwent tumor resection at our institution (63 males, 41 females). The 30-day tumor-related reoperation rate was $8 \%(8$ of 104 patients) ( $P=.002$ versus the iMRI cohort).

\section{DISCUSSION}

We have demonstrated that high-fieldstrength iMRI in children is safe; negates the need for sedation and postoperative MR imaging requiring travel from the safe confines of the intensive care unit; and markedly reduces the need for early reoperation by maximizing tumor resectability. The price of this technology is added operating room time, with an intraoperative scan adding approximately 45-60 minutes to a case, including the time for safety checks, patient preparation, moving the MR imaging scanner, and performing the scan. Performing stereotactic navigation sequences in the operating room before skin incision further prolongs operating room time. The preoperative preparation time, from entering the room to initial skin incision, was approximately 55 minutes longer when an intraoperative stereotactic scan was performed compared with use of a pre-existing scan. However, we have had no anesthesia or MR imaging-related complications, and our wound infection rates are no greater than those in historical controls. Continued follow-up is needed to assess the incidence of tumor recurrence, postoperative neurologic and cognitive deficits, and ultimately survival.

There are unique challenges in evaluating and interpreting intraoperative MR images. Gross changes in lesional signal inten-

AJNR Am J Neuroradiol 35:2382-87 Dec 2014 www.ajnr.org 
sity, diffusion characteristics, and enhancement patterns between the preoperative MR imaging and iMRI are often postoperative in nature rather than a reflection of residual tumor. For example, there are often thin areas of enhancement marginating the operative cavity (Fig 2), even in locations where there was no enhancing tumor on preoperative imaging. In most cases, these areas of enhancement most likely represent hyperemia/engorged vasculature and regional small-vessel permeability, especially if electrocautery (ie, bipolar) was performed. Intrinsic T1 shortening from blood products can be mistaken for enhancement if comparison with precontrast T1 imaging is not performed (Fig 2D), and subtraction imaging can help confirm this. Granulation tissue does not develop in the immediate intraoperative setting. Distinction of vascular enhancement from trace residual tumor may be difficult or impossible, particularly if the tumor shows marked preprocedural enhancement or if the enhancement is nodular in character. In equivocal cases, these areas should be explored intraoperatively or subject to higher scrutiny on follow-up examinations.

Multiple iterations of intraoperative scanning in patients with enhancing lesions requires repeat administration of intravenous gadolinium. Accordingly, care must be taken to ensure that there are no secondary signs of acute renal dysfunction, such as decreased urine output, to reduce the risk of nephrogenic systemic fibrosis. Use of half-dose $(0.05 \mathrm{mmol} / \mathrm{kg})$ contrast, possibly with high relaxivity and/or macrocyclic gadolinium chelates, may help mitigate this risk.

Little is known about the normal appearance of an active operative cavity after multiple contrast agent doses. The imaging appearance is complicated by the presence of an admixture of contrast material injected at 2 different time points. Theoretically, both false-positive and false-negative findings of residual tumor could be possible in patients who have received $>1$ contrast agent dose during a short time. Nonetheless, there have been no known instances of false-positive or false-negative tumor residual in our experience thus far.

For various reasons, image distortion may be present in patients undergoing iMRI. First, suboptimal patient positioning with respect to the magnet isocenter can result in spatial-resolution distortions. ${ }^{18}$ Paramagnetic susceptibility artifacts from blood products and gas can cause image distortion. These entities have major implications for iMRI. Even subtle differences between apparent and actual lesion location because of image distortion could alter the expected surgical outcome.

Performing an intraoperative MR imaging decreases the need for postoperative imaging and, in the case of CT, radiation exposure. Some patients without visible complications on the iMRI scan may be able to be followed in a step-down bed as opposed to a full intensive care unit bed. No repeat MR imaging scans were performed during the first week after resection in patients who had an iMRI after tumor resection.

Alternative intraoperative imaging modalities have been described, in particular sonography, however predominantly related to adult gliomas. ${ }^{19-23}$ Few uses of sonography have been shown in pediatric tumors. ${ }^{24,25}$ Sonography is highly operatordependent, and proper orientation with respect to the resection cavity can be difficult. iMRI allows spatial localization of the re- section cavity and possible residual tumor, in a manner that easily compares with preoperative imaging and can serve as a comparison study for postoperative follow-up scans.

In addition to brain tumor resection, iMRI may have other roles in the pediatric population. It can be used to confirm shunt placement, perhaps in a patient with multiloculated hydrocephalus; to confirm biopsy location in a suspected demyelinating disorder or vasculitis; in surgery for vascular lesions; and for confirmation of resection margins in epilepsy surgery. ${ }^{26-28}$

Success of high-field-strength iMRI requires careful attention to safety, and accordingly, patients with some implanted medical devices may not be able to safely undergo iMRI-guided surgery. Several patients at our institution were unable to undergo iMRIguided surgery because they had implanted devices that were not cleared for 3T scanning.

The large-bore scanner $(70 \mathrm{~cm})$ allowed patients to be scanned in various positions; however, upright surgery is not possible with this system. Additionally, the patient cannot fully enter the bore of the magnet, and tumors of the lower cervical spine and thoracic spine could not be imaged with current technology.

Successful implementation of an iMRI program requires collaboration, and accordingly, all cases at our institution are reviewed by the neuroradiologist and neurosurgeon before the operation to identify the goals of the operation and the intended approach. All iMRI scans are interpreted with the neuroradiologist in the operative suite at the time of scanning, providing realtime consultation with the neurosurgeon.

Future work will include outcome analysis looking at longterm disease-free survival and mortality. Additionally, a detailed cost-analysis will be helpful to account for the expenditures on the device itself and the increased operating room time.

\section{CONCLUSIONS}

In this series, the early tumor reoperation rate reduced from $8 \%$ to $<1 \%$ after instituting an iMRI program, which may result in reduced tumor recurrence and improved survival. If successful, an iMRI program can create a new paradigm for tumor treatment and establish the role for integrated real-time neuroradiology-neurosurgery collaboration for the resection of pediatric CNS tumors, which will translate into achieving optimal patient outcomes.

\section{REFERENCES}

1. Ostrom QT, Gittleman H, Farah P, et al. CBTRUS Statistical Report: primary brain and central nervous system tumors diagnosed in the United States in 2006-2010. Neuro Oncol 2013;15(suppl 2):ii1-ii56

2. Finlay JL, Wisoff JH. The impact of extent of resection in the management of malignant gliomas of childhood. Childs Nerv Syst 1999;15:786-88

3. Levy R, Cox RG, Hader WJ, et al. Application of intraoperative highfield magnetic resonance imaging in pediatric neurosurgery. $\mathrm{J} \mathrm{Neu}$ rosurg Pediatr 2009;4:467-74

4. Nimsky C, Ganslandt O, Von Keller B, et al. Intraoperative highfield-strength MR imaging: implementation and experience in 200 patients. Radiology 2004;233:67-78

5. Claus EB, Horlacher A, Hsu L, et al. Survival rates in patients with low-grade glioma after intraoperative magnetic resonance image guidance. Cancer 2005;103:1227-33

6. Knauth M, Wirtz CR, Tronnier VM, et al. Intraoperative MR imag- 
ing increases the extent of tumor resection in patients with highgrade gliomas. AJNR Am J Neuroradiol 1999;20:1642-46

7. Hall WA, Martin AJ, Liu H, et al. High-field strength interventional magnetic resonance imaging for pediatric neurosurgery. Pediatr Neurosurg 1998;29:253-59

8. Hall WA, Liu H, Martin AJ, et al. Safety, efficacy, and functionality of high-field strength interventional magnetic resonance imaging for neurosurgery. Neurosurgery 2000;46:632-41, discussion 641-42

9. Alexander E 3rd, Moriarty TM, Kikinis R, et al. The present and future role of intraoperative MRI in neurosurgical procedures. Stereotact Funct Neurosurg 1997;68(1-4 pt 1):10-17

10. Moriarty TM, Kikinis R, Jolesz FA, et al. Magnetic resonance imaging therapy; intraoperative MR imaging. Neurosurg Clin $N \mathrm{Am}$ 1996;7:323-31

11. Black PM, Alexander E, Martin C, et al. Craniotomy for tumor treatment in an intraoperative magnetic resonance imaging unit. $\mathrm{Neu}$ rosurgery 1999;45:423-31, discussion 431-33

12. Roth J, Beni Adani L, Biyani N, et al. Intraoperative portable 0.12-Tesla MRI in pediatric neurosurgery. Pediatr Neurosurg 2006;42:74-80

13. Black PM, Moriarty T, Alexander E, et al. Development and implementation of intraoperative magnetic resonance imaging and its neurosurgical applications. Neurosurgery 1997;41:831-42, discussion $842-45$

14. Samdani AF, Schulder M, Catrambone JE, et al. Use of a compact intraoperative low-field magnetic imager in pediatric neurosurgery. Childs Nerv Syst 2005;21:108-13, discussion 114

15. Souweidane MM. Intraoperative magnetic resonance imaging. J Neurosurg Pediatr 2009;4:465-66, discussion 466

16. Abernethy LJ, Avula S, Hughes GM, et al. Intra-operative 3-T MRI for paediatric brain tumours: challenges and perspectives. Pediatr Radiol 2012;42:147-57

17. Avula S, Mallucci CL, Pizer B, et al. Intraoperative 3-Tesla MRI in the management of paediatric cranial tumours: initial experience. $P e$ diatr Radiol 2012;42:158-67
18. Choudhri AF, Chin EM, Klimo P, et al. Spatial distortion due to field inhomogeneity in 3.0 Tesla intraoperative MRI. Neuroradiol J 2014;27:387-92

19. Hammoud MA, Ligon BL, elSouki R, et al. Use of intraoperative ultrasound for localizing tumors and determining the extent of resection: a comparative study with magnetic resonance imaging. J Neurosurg 1996;84:737-41

20. Regelsberger J, Lohmann F, Helmke K, et al. Ultrasound-guided surgery of deep seated brain lesions. Eur J Ultrasound 2000;12:115-21

21. Chen SY, Chiou TL, Chiu WT, et al. Application of intraoperative ultrasound for brain surgery. Tzu Chi Med J 2004;16:85-92

22. Cui LG, Jiang L, Zhang HB, et al. Monitoring of cerebrospinal fluid flow by intraoperative ultrasound in patients with Chiari I malformation. Clin Neurol Neurosurg 2011;113:173-76

23. Comeau RM, Fenster A, Peters TM. Intraoperative ultrasound in interactive neurosurgery. Radiographics 1998;18:1019-27

24. El Beltagy MA, Aggag M, Kamal M. Role of intraoperative ultrasound in resection of pediatric brain tumors. Childs Nerv Syst 2010; 26:1189-93

25. Ulrich NH, Burkhardt JK, Serra C, et al. Resection of pediatric intracerebral tumors with the aid of intraoperative real-time 3-D ultrasound. Childs Nerv Syst 2012;28:101-19

26. Roessler K, Sommer B, Grummich P, et al. Improved resection in lesional temporal lobe epilepsy surgery using neuronavigation and intraoperative MR imaging: favourable long term surgical and seizure outcome in 88 consecutive cases. Seizure 2014;23:201-07

27. Sommer B, Kasper BS, Coras R, et al. Surgical management of epilepsy due to cerebral cavernomas using neuronavigation and intraoperative MR imaging. Neurol Res 2013;35:1076-83

28. Sun GC, Chen XL, Zhao Y, et al. Intraoperative MRI with integrated functional neuronavigation-guided resection of supratentorial cavernous malformations in eloquent brain areas. J Clin Neurosci 2011;18:1350-54 